Revista PSICOLOGIA, 2020, Vol. 34 (1), 121-134. doi: 10.17575/psicologia.v34i1.1496

\title{
Impacto dos fatores psicossociais de risco na qualidade de vida da população no processo de reforma
}

\author{
Tania Gaspar1,2,5, Diego Gomez-Baya ${ }^{3}$, Isabel Torres ${ }^{4}$, Ana Cerqueira1,5, Manuela Faia \\ Correia $^{1}$ \& Margarida Gaspar de Matos $^{2,5}$ \\ ${ }^{1}$ Universidade Lusíada de Lisboa/CLISSIS/ COMEGI \\ ${ }^{2}$ Universidade de Lisboa/FML/ISAMB \\ ${ }^{3}$ Universidade de Huelva/ Departamento de Psicologia Social, Desenvolvimento e Educacional \\ ${ }^{4}$ Universidade Lusíada do Porto/CLISSIS \\ ${ }^{5}$ Universidade de Lisboa/FMH/Aventura Social
}

\begin{abstract}
Resumo: O objetivo principal deste estudo é compreender e caracterizar a relação entre os fatores de risco psicossocial relacionados ao trabalho e as dimensões de qualidade de vida (QV) em adultos profissionalmente ativos e reformados em Portugal. Os dados foram recolhidos ao nível nacional. Participaram 1.330 indivíduos, sendo 62,2\% do género feminino, com idades entre 55 e 75 anos. Foram analisadas as diferenças de género, idade e situação profissional (profissional ativo e reformado) em relação aos fatores psicossocial de risco e QV. Para analisar esta relação foram testados cinco modelos de regressão. Os resultados mostraram que a gestão do stress/burnout e a sobrecarga de trabalho são os fatores de risco psicossocial que apresentaram um impacto mais forte na qualidade de vida. 0 estudo permitiu concluir que a QV no processo de reforma é influenciada pelos riscos psicossociais relacionados com o trabalho, principalmente a gestão do stress e a sobrecarga de trabalho.
\end{abstract}

Palavras-chave: Fatores de risco psicossociais relacionados ao trabalho; gestão do stress; qualidade de vida; situação de trabalho; saúde física e psicológica.

Impact of psychosocial risk factors on the population's quality of life in the retirement process. The main objective of this study is to better understand and characterize the relationship between work-related psychosocial risk factors and quality of life dimensions in working and retired population. The data were collected at national level in Portugal. Participants were 1,330 persons, $62.2 \%$ of which female, with ages ranging between 55 and 75 years old. Were analyzed gender, age and professional status (professional active and retired) differences in relation to psychosocial risk factors and quality of life. Five regressions models were tested. Results showed that the stress/burnout management and work overload are the psychosocial risk factors that presented a strong impact in quality of live. Quality of live dimensions. Our study allowed to conclude that quality of life in retirement and aging process is influenced by work-related psychosocial risks, especially stress management and work overload.

Keywords: Work-related psychosocial risks factors; stress management, quality of life, working status, physical and psychological health.

O envelhecimento da população acarreta mudanças e consequências a um nível individual e global, refletindo-se na sociedade em geral (Illario et al., 2016; Mari et al., 2016; World Health Organization [WHO], 2015). De acordo com o INE, Portugal caracteriza-se pelo o decréscimo da população jovem e da população em idade ativa, a par do aumento da população idosa, em resultado da queda da natalidade e do aumento da longevidade nos últimos anos. De acordo com as projeções nacionais, prevê-se que, em 2030, os idosos representem cerca de 26\% da população e, em 2060, cresçam para 29\%. A esperança média de vida aos 65 anos em Portugal ultrapassava os 19 anos (perto de 21 anos no caso das mulheres e 17 anos para os homens), já no que respeita ao indicador que mede a esperança de vida saudável aos 65 anos, o ano mais recente com informação estatística para Portugal refere-se a 2014 e apresenta para as mulheres 5,6 anos após os 65 em que pode esperar viver em condições de vida saudável e os homens 6,9 anos após os 65 . 0 envelhecimento da população residente em Portugal contribuiu determinantemente para a evolução da estrutura do mercado de trabalho. Neste sentido, os indivíduos com 55 e mais anos representavam 19,5\% do total da população ativa, sendo que $14,8 \%$ se situava entre os 55 e os 64 anos. 0 trabalho proporciona

1 Morada para correspondência: Rua da Junqueira no188-198 1340-001 Lisboa. E-mail: tania.gaspar.barra@gmail.com. 
quer uma atividade estruturada dentro de um quadro temporal, um sentimento de propósito e é também fonte de significado A reforma é um acontecimento de vida muito relevante que normalmente acorre no processo de envelhecimento. A reforma implica a retirada (de forma mais ou menos progressiva) do mundo do trabalho (Byles et al., 2013), o que se pode refletir ao nível do bem-estar e qualidade de vida (QV), devido ao afastamento do local de trabalho e dos colegas e, também, ao surgimento de sentimentos negativos como, a diminuição da autoestima, ansiedade e depressão. Para além disso, nesta fase assiste-se a um aumento no risco de aparecimento de doenças crónicas e de dificuldades em realizar as atividades diárias, tendendo a associar-se a piores resultados ao nível da saúde física e mental dos indivíduos (Behncke, 2012). Um ajustamento positivo é essencial para manter o bem-estar físico e psicológico mais tarde na vida. (Wong \& Earl,2009), proporcionando conforto face à vida na reforma (Wang et al., 2011).

Existem evidências na literatura relativamente à reforma ser, só por si, um evento causador de stress (Coe \& Zamarro, 2011; Fonseca, 2012). Nos casos em que a reforma é encarada como a perda de contactos, de relacionamentos interpessoais e de valores intrínsecos, esta mudança pode aumentar os níveis de stress (van der Heide et al., 2013), comparativamente com aqueles vivenciados antes deste processo. Os reformados também perdem a sua identidade social e valor próprio derivados do trabalho. De acordo com Wang et al. (2011), os recursos físicos, financeiros, sociais, emocionais, cognitivos e motivacionais tendem a diminuir depois da reforma. No entanto, alguns investigadores defendem que a reforma não é necessariamente traumática ou stressante (e.g., Kim \& Moen, 2002; Fehr, 2012), e nem toda a experiência de reformado diminui o seu bem-estar físico e psicológico (Wang, 2007).A QV é um estado relacionado ao bem-estar físico, mental e social, (WHO, 2002) e é um conceito que engloba diversos aspetos, tais como a saúde física, mental, o estado psicológico (cognitivo e emocional), as relações sociais e laborais, a condição económica e as atividades recreativas e de lazer. Assim, a sua avaliação baseia-se em indicadores objetivos e/ou subjetivos relativos a aspetos físicos, cognitivos, emocionais e sociais (Efklides \& Moraitou, 2013; Halvorsrud \& Kalfoss, 2007).

O bem-estar dos indivíduos é claramente influenciado pela condição de saúde, mas também pelas condições financeiras, as relações interpessoais, o desempenho de papéis e as atividades sociais (Steptoe, Deaton \& Stone, 2015) e reflete-se ao nível da qualidade de vida dos indivíduos (Friedman, et al., 2017). Os estilos de vida, o ambiente no qual os indivíduos se inserem e a inclusão social têm emergido como fatores relevantes que condicionam o envelhecimento (European Commission, 2015, 2018; Illario, et al., 2016).

A atividade laboral e os fatores psicossociais relacionados com esse ambiente constituem-se como aspetos importantes para a qualidade de vida dos indivíduos (Helkavaara et al., 2011; Jönsson, 2012; Nieuwenhuijsen et al., 2010; Theorell et al., 2015). No seu dia a dia, os trabalhadores estão expostos a diversas condições potencialmente prejudiciais para a sua QV (Burgard \& Lin, 2013), sendo que quanto maior é o risco psicossocial do ambiente laboral, maior será o prejuízo ao nível da qualidade de vida física e psicológica, presente e futura (Bonde, 2008; Eatough et al., 2012; Nieuwenhuijsen et al., 2010).

Com o aumento da idade surgem alguns estereótipos face aos trabalhadores mais velhos que tendem a relacioná-los com uma menor capacidade de trabalho, de aprendizagem e de crescimento. Assim, transparecem algumas assunções, nomeadamente a de estarem à espera da reforma, de serem mais resistentes ao uso das novas tecnologias, menos aptos para a aprendizagem, mais lentos no processamento de informação, mais inflexíveis, menos motivados, entre outros aspetos (Gaillard \& Desmette, 2010; Malinen \& Johnston, 2013; Truxillo et al., 2012). Estas ideias e assunções negativas que surgem em torno dos trabalhadores mais velhos, podem constituir-se como um fator de stress, na medida em que se refletem nos fatores relacionados com o trabalho e podem condicionar diversos aspetos da sua vida laboral (Kulik et al., 2016; Rabl, 2010). Quando efetivamente estas ideias estão presentes no ambiente laboral, isso pode conduzir a prejuízos na experiência e desempenho das tarefas (Adams et al., 2006; Smith et al., 2007) e a uma redução significativa ao nível da confiança nas suas próprias capacidades (Koch et al., 2008).

Nos casos em que os indivíduos ainda se encontram com atividade profissional ativa, existem diferentes fontes de stress presentes no contexto laboral, como por exemplo, o fraco suporte e/ou conflitos com colegas e/ou chefias, os ambientes de elevada exigência, relação família/trabalho (Khamisa et al., 2015; Nieuwenhuijsen et al., 2010), que trazem inúmeras repercussões para a saúde física e mental dos indivíduos (Teles et al., 2014).

0 presente estudo pretende compreender e caracterizar a relação entre os riscos psicossociais e a qualidade de vida e respetivas dimensões. Pretende-se, ainda, estudar o impacto do género, idade e situação profissional dos participantes nesta relação. 


\section{MÉTODO}

\section{Participantes}

Participaram no presente estudo um total de 1330 indivíduos com idades entre os 55 e os 75 anos, residentes em Portugal continental, sendo que $62,2 \%$ são do género feminino. Em relação à situação profissional, 47\% têm atividade profissional ativa, 46,5\% são reformados e 6,5\% são reformados, mas com atividade profissional ativa. Quanto ao estado civil, 64,8\% são casados ou unidos de facto e 35,2\% referem

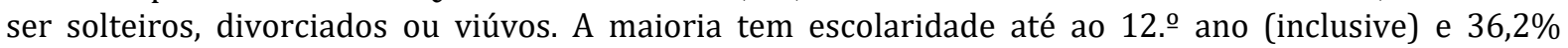
completou o ensino superior, mestrado ou doutoramento. Trata-se de uma amostra não probabilística, por conveniência.

\section{Instrumento}

O instrumento foi composto por questões sociodemográficas, e por instrumentos de avaliação relacionados com a qualidade de vida e fatores psicossociais.

Qualidade de Vida (WHOQOL-BREF). 0 nome do instrumento é WHOQOL-BREF (World Health Organization Quality of Life - Bref), os autores e a versão original são do Grupo WHOQOL, 1994 (Grupo de Qualidade de Vida da Organização Mundial da Saúde) e neste estudo foi utilizada a versão portuguesa (Canavarro, et al., 2007). É uma medida genérica, multidimensional e multicultural para avaliar a qualidade de vida subjetiva. É composto por 26 itens e inclui quatro domínios de qualidade de vida: Físico "Tem energia suficiente para a sua vida diária?", Psicológico “Até que ponto está satisfeito(a) consigo", Social “Até que ponto está satisfeito(a) com o apoio que recebe dos seus amigos?" e Ambiental "Até que ponto está satisfeito(a) com o acesso que tem aos serviços de saúde?". Cada um desses domínios consiste em facetas de qualidade de vida. Esta medida, também, permite o cálculo de um indicador global da qualidade de vida. Os itens são avaliados numa escala do tipo Likert de 5 pontos: 1 - muito má / muito insatisfeito / nada / nunca a 5 - muito mau/muito insatisfeito/ completamente /sempre.

A consistência interna, medida pelo alfa de Cronbach, mostra valores aceitáveis, seja analisando os quatro domínios $(0,90)$ ou cada domínio individual variando de 0,86 (Ambiental) e 0,95 (Psicológico).

Fatores psicossociais do trabalho (COPSOQ). 0 instrumento utilizado para medir os fatores psicossociais no trabalho (Copenhagen Psychosocial Questionnaire - COPSOQ II), foi desenvolvido e validado por Kristensen et al. (2005) em colaboração com o Instituto Nacional Dinamarquês de Saúde Ocupacional em Copenhaga. 0 seu objetivo principal é avaliar os fatores psicossociais no trabalho. 0 COPSOQ II segue um conceito multidimensional e destina-se a incluir as necessidades gerais envolvidas na base do conceito de "stress no trabalho". Pretende explicar os fatores de risco psicossocial no trabalho, como resultado de altas exigências de trabalho e baixo suporte social. Neste estudo foi utilizada a versão em portuguesa (Silva, et al., 2011), mas foi retirada a escala de violência no trabalho porque é uma questão muito sensível e numa perspetiva ética não foi considerada uma variável fundamental para o presente estudo. Esta versão resumida inclui as dimensões psicossociais com evidências epidemiológicas relacionadas à saúde. Todas as versões avaliam indicadores de exposição (riscos psicossociais) e indicadores de seus efeitos (saúde, satisfação e stress). Todos os itens das três versões são avaliados numa escala do tipo Likert de 5 pontos: 1 - nunca / quase nunca a 5 - sempre/quase sempre. Através da Análise Fatorial Exploratória com rotação varimax foram identificadas seis dimensões com uma variância explicada total de 69,85\%. De seguida, serão apresentadas as dimensões identificadas, consistência interna (alfa de Cronbach) e exemplos ilustrativos dos respetivos itens e : Gestão Stress/ Burnout $(\alpha=0,90)$ "Com que frequência se sente emocionalmente exausto?"; Sobrecarga de Trabalho $(\alpha=0,86)$ "Com que frequência não tem tempo para completar todas as tarefas do seu trabalho?"; Autonomia $(\alpha=0,82)$ "Tem um nível elevado de influência sobre o seu trabalho?"; Transparência de papel $(\alpha=0,60)$ "Sabe exatamente o que é esperado do seu trabalho?"; Suporte chefia $(\alpha=0,58)$ "Costuma ter ajuda e apoio do seu superior direto?"; Confiança/Justiça $(\alpha=0,56)$ "É tratado de forma justa no seu local de trabalho?".

\section{Procedimento}

Foram contactadas diversas organizações, como sindicatos, empresas, municípios, universidades seniores, e Instituições Particulares de Solidariedade Social (IPSS) que incluíam pessoas na faixa etária do estudo. Ou seja, pessoas com idades entre os 55 e os 75 anos, dez anos antes e dez anos depois da idade oficial de reforma. A recolha de dados foi realizada com as instituições que concordaram em cooperar e com as pessoas que concordaram em preencher o questionário. 0 questionário foi de autopreenchimento, anónimo e confidencial. Relativamente aos participantes reformados foi-lhes pedido que reportassem as suas respostas ao último ano de trabalho. 
O projeto foi aprovado pela comissão de ética da ARSLVT / Ministério da Saúde pro. 023 / CES / INV / 2014.

\section{RESULTADOS}

Na Tabela 1 apresentam-se as médias e consistência interna das dimensões das escalas em estudo, a maioria das subescalas apresenta bons valores de consistência interna, com exceção de três dimensões dos fatores psicossociais de trabalho que apresentam valores menos adequados, nomeadamente, na dimensão transparência, Suporte da chefia e Confiança e justiça que apresentam valores entre os .60 e .56.

Tabela 1. Medidas descritivas e consistência interna (alfa de Cronbach) dos fatores psicossociais de risco e qualidade de vida

\begin{tabular}{lccc}
\hline & Média & Desvio Padrão & $\alpha$ \\
\hline Stress/Burnout & 3.21 & 0.80 & .90 \\
Sobrecarga & 2.75 & 0.72 & .86 \\
Autonomia & 2.40 & 0.94 & .82 \\
Transparência & 2.25 & 0.83 & .60 \\
Suporte chefia & 2.50 & 0.90 & .58 \\
Confiança/Justiça & 2.30 & 0.87 & .56 \\
Qualidade de Vida geral & 3.67 & 0.69 & .90 \\
Qualidade de Vida física & 3.88 & 0.68 & .94 \\
Qualidade de Vida psicológica & 3.90 & 0.60 & .95 \\
Qualidade de Vida social & 3.85 & 0.62 & .85 \\
Qualidade de Vida ambiental & 3.48 & 0.59 & .86 \\
\hline
\end{tabular}

Através da análise da Tabela 2, é possível verificar que existem correlações entre todas as variáveis, com exceção da sobrecarga de trabalho com a baixa transparência de papel e com a QV geral. Assim, pode observar-se que existe uma forte relação entre as dimensões dos fatores psicossociais e as dimensões da qualidade de vida. 
Tabela 2. Correlações entre fatores psicossociais de risco e qualidade de vida

\begin{tabular}{|c|c|c|c|c|c|c|c|c|c|c|}
\hline Variáveis & 1 & 2 & 3 & 4 & 5 & 6 & 7 & 8 & 9 & 10 \\
\hline Gestão Stress / Burnout (1) & -- & & & & & & & & & \\
\hline Sobrecarga de trabalho (2) & $-0.33^{* * *}$ & -- & & & & & & & & \\
\hline Baixa Autonomia (3) & $-0.07^{*}$ & $-0.10^{* * *}$ & -- & & & & & & & \\
\hline Baixa Transparência de papel (4) & $-0.13^{* * *}$ & 0.06 & $0.39 * * *$ & -- & & & & & & \\
\hline Baixo Suporte chefia (5) & $-0.15^{* * *}$ & $0.17^{* * *}$ & $0.20^{* * *}$ & $0.63^{* * *}$ & -- & & & & & \\
\hline Baixa Confiança/ Justiça (6) & $-0.20^{* * *}$ & $0.19^{* * *}$ & $0.17^{* * *}$ & $0.54^{* * *}$ & $0.64^{* * *}$ & -- & & & & \\
\hline Qualidade de Vida geral (7) & $0.39 * * *$ & 0.02 & $-0.16^{* * *}$ & $-0.21^{* * *}$ & $-0.12^{* * *}$ & $-0.13^{* * *}$ & -- & & & \\
\hline Qualidade de Vida física (8) & $0.48^{* * *}$ & $-0.86^{* *}$ & $-0.22^{* * *}$ & $-0.23^{* * *}$ & $-0.12^{* * *}$ & $-0.17^{* * *}$ & $0.67^{* * *}$ & -- & & \\
\hline Qualidade de Vida psicológica (9) & $0.50^{* * *}$ & $-0.08^{* *}$ & $-0.25^{* * *}$ & $-0.27^{* * *}$ & $-0.18^{* * *}$ & $-0.23^{* * *}$ & $0.58^{* * *}$ & $0.67^{* * *}$ & -- & \\
\hline Qualidade de Vida social (10) & $0.33^{* * *}$ & $0.09^{* *}$ & $-0.13^{* * *}$ & $-0.21^{* * *}$ & $-0.19 * * *$ & $-0.24 * * *$ & $0.40^{* * *}$ & $0.49^{* * *}$ & $0.55^{* * *}$ & -- \\
\hline Qualidade de Vida ambiental (11) & $0.39 * * *$ & $-0.06^{*}$ & $-0.14^{* * *}$ & $-0.28^{* * *}$ & $-0.22^{* * *}$ & $-0.22^{* * *}$ & $0.60^{* * *}$ & $0.65^{* * *}$ & $0.58^{* * *}$ & $0.42^{* * *}$ \\
\hline
\end{tabular}

Nota. ${ }^{* * *} p<0.001 ;{ }^{* *} p<0.01 ;{ }^{*} p<0.05$ 
Através dos resultados obtidos (Tabela 3) pode observar-se que existem diferenças significativas no que diz respeito ao género, na dimensão gestão de stress/burnout, baixa confiança/justiça, QV física, psicológica e ambiental, sendo o género masculino aquele que apresenta médias mais elevadas nestas dimensões, com exceção da perceção de baixa confiança/justiça, na qual é o género feminino que apresenta um valor mais elevado.

Tabela 3. ANOVA - Diferenças entre as dimensões dos fatores psicossociais de risco qualidade de vida e género

\begin{tabular}{|c|c|c|c|c|c|}
\hline \multirow[b]{2}{*}{ Dimensões } & \multicolumn{2}{|c|}{ Feminino } & \multicolumn{2}{|c|}{ Masculino } & \multirow{2}{*}{$\boldsymbol{F}$} \\
\hline & $M$ & $D P$ & $M$ & $D P$ & \\
\hline Gestão Stress/Burnout & 3.14 & 0.79 & 3.35 & 0.81 & $19.20 * * *$ \\
\hline Sobrecarga de trabalho & 2.77 & 0.71 & 2.71 & 0.72 & 1.77 \\
\hline Baixa Autonomia & 2.44 & 0.94 & 2.34 & 0.96 & 2.95 \\
\hline Baixa Transparência de papel & 2.27 & 0.83 & 2.20 & 0.83 & 1.85 \\
\hline Baixo Suporte chefia & 2.53 & 1.06 & 2.46 & 0.16 & 1.06 \\
\hline Baixa Confiança/Justiça & 2.35 & 0.86 & 2.22 & 0.89 & $5.51 *$ \\
\hline Qualidade de Vida geral & 3.65 & 0.71 & 3.71 & 0.67 & 2.44 \\
\hline Qualidade de Vida física & 3.83 & 0.70 & 3.98 & 0.64 & $13.02^{* * *}$ \\
\hline Qualidade de Vida psicológica & 3.85 & 0.61 & 3.99 & 0.57 & $16.83^{* * *}$ \\
\hline Qualidade de Vida social & 3.85 & 0.63 & 3.84 & 0.63 & 0.03 \\
\hline Qualidade de Vida ambiental & 3.45 & 0.59 & 3.54 & 0.59 & $5.24^{*}$ \\
\hline
\end{tabular}

Nota. $^{* * *} p<.001 ;{ }^{* *} p<.01$

Relativamente à idade (Tabela 4), podem observar-se diferenças estatisticamente significativas nas seguintes dimensões: gestão de stress/burnout, sobrecarga de trabalho, QV geral, física, psicológica e social. Assim, verifica-se que são os indivíduos até aos 60 anos que apresentam valores mais elevados de sobrecarga de trabalho e de QV geral, física, psicológica e relativa às relações sociais. Por outro lado, são os indivíduos com 61 ou mais anos que apresentam menores níveis de stress/burnout.

Tabela 4. ANOVA - Diferenças entre as dimensões dos fatores psicossociais de risco, qualidade de vida e idade

\begin{tabular}{|c|c|c|c|c|c|}
\hline \multirow{2}{*}{ Dimensões } & \multicolumn{2}{|c|}{ Até 60 anos } & \multicolumn{2}{|c|}{61 ou mais anos } & \multirow{2}{*}{$\boldsymbol{F}$} \\
\hline & $M$ & $D P$ & $M$ & $D P$ & \\
\hline Gestão Stress/Burnout & 3.14 & 0.80 & 3.29 & 0.79 & $11.26^{* * *}$ \\
\hline Sobrecarga de trabalho & 2.87 & 0.69 & 2.64 & 0.72 & $33.07^{* * *}$ \\
\hline Baixa Autonomia & 2.37 & 0.87 & 2.44 & 1.00 & 1.84 \\
\hline Baixa Transparência de papel & 2.26 & 0.82 & 2.23 & 0.84 & 0.41 \\
\hline Baixo Suporte chefia & 2.53 & 1.12 & 2.47 & 1.00 & 0.87 \\
\hline Baixa Confiança/ Justiça & 2.31 & 0.89 & 2.29 & 0.86 & 0.18 \\
\hline Qualidade de Vida geral & 3.73 & 0.67 & 3.62 & 0.70 & $7.37^{* *}$ \\
\hline Qualidade de Vida física & 3.95 & 0.67 & 3.82 & 0.69 & $10.34^{* * *}$ \\
\hline Qualidade de Vida psicológica & 3.94 & 0.59 & 3.87 & 0.60 & $3.83^{*}$ \\
\hline Qualidade de Vida social & 3.90 & 0.63 & 3.81 & 0.61 & $6.16^{* *}$ \\
\hline Qualidade de Vida ambiental & 3.47 & 0.60 & 3.49 & 0.59 & 0.32 \\
\hline
\end{tabular}

Nota. ${ }^{* * *} p<0.001 ;{ }^{* *} p<.01 ;{ }^{*} p<.05$

Os resultados da Tabela 5 indicam diferenças estatisticamente significativas entre as diferentes situações profissionais para todas as variáveis com exceção da dimensão referente à baixa transparência de papel. Deste modo, verifica-se que as dimensões dos fatores psicossociais (com exceção da referida) e da qualidade de vida variam consoante a situação profissional. São os participantes reformados com atividade profissional ativa que apresentam uma melhor perceção de qualidade de vida geral, qualidade de vida física e psicológica, assim como melhor gestão de stress/burnout e maior autonomia. Os participantes reformados apresentam uma perceção mais negativa em relação ao suporte da chefia, confiança e justiça 
remetendo-se ao seu último ano de atividade profissional. Também são os praticantes reformados que referem uma perceção de QV geral mais negativa. Os participantes com atividade profissional ativa apresentam menor capacidade de gestão do stress/burnout, maior sobrecarga de trabalho, melhor perceção de qualidade de vida social e ambiental.

Tabela 5. ANOVA - Diferenças entre as dimensões dos fatores psicossociais, qualidade de vida e situação profissional

\begin{tabular}{lccccccc}
\hline \multirow{2}{*}{ Dimensões } & \multicolumn{2}{c}{$\begin{array}{c}\text { Atividade } \\
\text { profissional ativa }\end{array}$} & Reformado(a) & \multicolumn{2}{c}{$\begin{array}{c}\text { Reformado(a) } \\
\text { com atividade } \\
\text { profissional ativa }\end{array}$} & $\boldsymbol{F}$ \\
\cline { 2 - 6 } & $\boldsymbol{M}$ & $\boldsymbol{D P}$ & $\boldsymbol{M}$ & $\boldsymbol{D P}$ & $\boldsymbol{M}$ & $\boldsymbol{D P}$ & \\
\hline Gestão Stress/Burnout & 3.14 & 0.79 & 3.25 & 0.79 & 3.51 & 0.91 & $\mathbf{8 . 1 4}^{* * *}$ \\
Sobrecarga de trabalho & 2.87 & 0.68 & 2.67 & 0.72 & 2.44 & 0.71 & $\mathbf{1 7 . 9 5}^{* * *}$ \\
Baixa Autonomia & 2.36 & 0.87 & 2.47 & 1.02 & 2.16 & 0.99 & $\mathbf{4 . 0 7}^{*}$ \\
Baixa Transparência de papel & 2.27 & 0.81 & 2.22 & 0.83 & 2.08 & 0.89 & 2.01 \\
Baixo Suporte chefia & 2.49 & 1.01 & 2.12 & 0.98 & 2.49 & 1.06 & $\mathbf{4 . 4 0}^{*}$ \\
Baixa Confiança/ Justiça & 2.29 & 0.87 & 2.32 & 0.85 & 2.04 & 0.92 & $\mathbf{3 . 1 3}^{*}$ \\
Qualidade de Vida geral & 3.73 & 0.67 & 3.58 & 0.71 & 3.85 & 0.62 & $\mathbf{9 . 8 3}^{* * *}$ \\
Qualidade de Vida física & 3.95 & 0.65 & 3.76 & 0.70 & 4.24 & 0.64 & $\mathbf{2 1 . 3 0}^{* * *}$ \\
Qualidade de Vida psicológica & 3.92 & 0.58 & 3.85 & 0.61 & 4.16 & 0.54 & $\mathbf{9 . 0 0}^{* * *}$ \\
Qualidade de Vida social & 3.93 & 0.61 & 3.78 & 0.63 & 3.86 & 0.60 & $\mathbf{8 . 6 4}^{* * *}$ \\
Qualidade de Vida ambiental & 3.86 & 0.59 & 3.49 & 0.57 & 3.69 & 0.67 & $\mathbf{5 . 1 1}^{* *}$ \\
\hline
\end{tabular}

Nota. ${ }^{* * *} p<.001 ;{ }^{* *} p<.01 ;{ }^{*} p<.05$

De forma a compreender e explicar o impacto dos fatores psicossociais nas diferentes áreas da qualidade de vida, foram realizados cinco modelos de regressão linear múltipla para cada uma das situações profissionais em estudo (reformados, profissionais ativos e reformados com atividade profissional), que incluem variáveis sociodemográficas (género, idade, estado civil e escolaridade) e variáveis relacionadas com os fatores psicossociais no trabalho (Tabela 6, 7 e 8).

Em relação aos indivíduos com situação profissional ativa (Tabela 6), de uma forma geral e em relações às variáveis sociodemográficas, salienta-se que o género não se relaciona significativamente com nenhuma das dimensões da QV, a idade apenas surge como variável explicativa da QV física, o estado civil (solteiro/divorciado/viúvo) associa-se de forma negativa e significativa com as dimensões da QV, com exceção da referente à física e, por último, a escolaridade relaciona-se com a $\mathrm{QV}$ em todas as dimensões menos na social. No que diz respeito aos fatores psicossociais, a dimensão da gestão do stress/burnout relaciona-se de forma positiva e significativa com todas as dimensões da QV (maior valor, menos stress) e a dimensão que reflete baixa transparência de papel apresenta uma relação negativa com todas as dimensões da QV à exceção da social.

De acordo com o primeiro modelo (QV geral), verifica-se que a escolaridade, a sobrecarga de trabalho e a gestão do stress/burnout se associam de forma positiva e significativa com a QV geral. 0 estado civil (solteiro/divorciado/viúvo), a baixa autonomia e a baixa transparência de papel, por sua vez, têm uma associação negativa e significativa com esta dimensão. No seu conjunto, o poder explicativo do modelo 1 é de $28 \%$ da variância, $F(10.439)=18.28 ; p<0.001$.

Por sua vez, no segundo modelo (QV física), as variáveis idade, escolaridade, baixa autonomia, baixa transparência de papel, baixo suporte da chefia, baixa confiança/justiça e gestão do stress/burnout surgem como explicativas da QV física. Este modelo apresenta um poder explicativo de $35 \%$ da variância, $F(10.428)$ $=24.64 ; p<0.001$.

No terceiro modelo (QV psicológica), as variáveis que surgem associadas com esta dimensão são o estado civil, a escolaridade, a baixa autonomia, a baixa transparência de papel, a baixa confiança/justiça e a gestão do stress/burnout. 0 modelo 3 explica $36 \%$ da variância, $F(10.432)=25.78 ; p<0.001$.

$\mathrm{O}$ quarto modelo (QV social) permite observar que apenas as variáveis estado civil, baixa autonomia, baixa confiança/justiça e gestão do stress/burnout surgem como explicativas da QV social. No seu conjunto, o poder explicativo deste modelo é de $17 \%$ da variância, $F(10.444)=10.50 ; p<0.001$, sendo o modelo que apresenta o menor poder explicativo. 
Por último, no modelo cinco (QV ambiental), o estado civil, a escolaridade, a baixa transparência de papel e a gestão do stress/burnout surgem significativamente associados à QV ligada ao ambiente. Este modelo apresenta um poder explicativo de $36 \%$ da variância, $F(10.416)=24.81 ; p<0.001$.

Tabela 6. Modelos de regressão linear múltipla para o estudo da qualidade de vida nos indivíduos com atividade profissional ativa

\begin{tabular}{|c|c|c|c|c|c|}
\hline & $\begin{array}{c}\text { Modelo } 1 \\
\text { QV geral } \\
\end{array}$ & $\begin{array}{l}\text { Modelo } 2 \\
\text { QV física } \\
\end{array}$ & $\begin{array}{c}\text { Modelo } 3 \\
\text { QV psicológica } \\
\end{array}$ & $\begin{array}{l}\text { Modelo } 4 \\
\text { QV social } \\
\end{array}$ & $\begin{array}{c}\text { Modelo 5 } \\
\text { QV ambiental } \\
\end{array}$ \\
\hline & $\boldsymbol{\beta}$ & $\boldsymbol{\beta}$ & $\boldsymbol{\beta}$ & $\boldsymbol{\beta}$ & $\boldsymbol{\beta}$ \\
\hline Género**** & -0.00 & 0.07 & 0.07 & -0.02 & 0.04 \\
\hline Idade & 0.05 & $-0.08^{*}$ & -0.06 & -0.01 & 0.03 \\
\hline Estado civil ${ }^{* * * * *}$ & $-0.11^{* *}$ & -0.03 & $-0.14^{* * *}$ & $-0.10^{*}$ & $-0.08^{*}$ \\
\hline Escolaridade & $0.23^{* * *}$ & $0.27 * * *$ & $0.18 * * *$ & 0.06 & $0.38^{* * *}$ \\
\hline Sobrecarga de trabalho & $0.13^{* *}$ & -0.03 & 0.07 & 0.03 & -0.01 \\
\hline Baixa Autonomia & $-0.08 *$ & $-0.18 * * *$ & $-0.13^{* * *}$ & $-0.12^{* *}$ & -0.04 \\
\hline Baixa Transparência de papel & $-0.16^{* *}$ & $-0.16^{* *}$ & $-0.14^{* *}$ & -0.08 & $-0.19 * * *$ \\
\hline Baixo Suporte chefia & 0.07 & $0.25^{* * *}$ & 0.05 & 0.07 & 0.01 \\
\hline Baixa Confiança/ Justiça & -0.05 & $-0.15^{* *}$ & $-0.15^{* *}$ & $-0.21 * * *$ & -0.08 \\
\hline Gestão Stress/Burnout & $0.39 * * *$ & $0.38^{* * *}$ & $0.41^{* * *}$ & $0.29 * * *$ & $0.31^{* * *}$ \\
\hline
\end{tabular}

Nota. ${ }^{* * *} p<.001 ;{ }^{* *} p<.01 ;{ }^{*} p<.05 ;$ n.s. = não significativo; ${ }^{* * *}\left(1-\right.$ Feminino; 2 - Masculino); ${ }^{* * * * *}(1-$ Casado/união facto; 2 - solteiro/divorciado)

No que diz respeito aos reformados (Tabela 7), pode observar-se que o estado civil (solteiro/divorciado/viúvo) se relaciona de forma negativa e significativa com todas as dimensões da QV e a escolaridade de forma positiva e significativa com todas, à exceção da QV social. No que diz respeito aos fatores psicossociais, e tal como acontece no caso dos indivíduos com atividade profissional ativa, a dimensão de gestão do stress/burnout evidencia uma relação positiva e significativa com todas as dimensões da QV (maior valor, menos stress) e a dimensão da baixa transparência de papel apresenta uma relação negativa com todas as dimensões da QV à exceção da social.

No primeiro modelo é possível verificar que o estado civil, a escolaridade, a baixa transparência de papel e a gestão do stress/burnout se relacionam com a QV geral. No seu conjunto, o poder explicativo do modelo 1 é de $26 \%$ da variância, $F(10.353)=13.94 ; p<0.001$.

No modelo referente à QV física, as variáveis: idade, estado civil, escolaridade, baixa transparência de papel e gestão do stress/burnout surgem como explicativas desta dimensão. Este modelo apresenta um poder explicativo de $39 \%$ da variância, $F(10.352)=23.78 ; p<0.001$.

0 terceiro modelo (QV psicológica) permite observar que, nos fatores sociodemográficos, apenas o género não se relaciona com esta dimensão da QV. No que diz respeito aos fatores psicossociais, aqueles que surgem como explicativos são a falta de autonomia, a falta de transparência de papel e gestão do stress/burnout. Este modelo explica $43 \%$ da variância, $F(10.350)=27.82 ; p<0.001$.

0 modelo da QV social demonstra que apenas o género, estado civil e a gestão do stress/burnout se constituem como variáveis explicativas desta dimensão da QV. No seu conjunto, o poder explicativo deste modelo é de $21 \%$ da variância, $F(10.343)=10.24 ; p<0.001$.

Em relação ao modelo cinco (QV ambiental), que explica $31 \%$ da variância, $F(10.348)=17.00 ; p<$ 0.001, pode observar-se que as variáveis sociodemográficas com exceção da idade, a sobrecarga de trabalho, a baixa transparência de papel e gestão do stress/burnout surgem significativamente associados à QV ligada ao ambiente. 
Tabela 7. Modelos de regressão linear múltipla para o estudo da qualidade de vida nos indivíduos reformados

\begin{tabular}{|c|c|c|c|c|c|}
\hline & $\begin{array}{c}\text { Modelo } 1 \\
\text { QV geral }\end{array}$ & $\begin{array}{c}\text { Modelo } 2 \\
\text { QV física }\end{array}$ & $\begin{array}{c}\text { Modelo 3 } \\
\text { QV } \\
\text { psicológica } \\
\end{array}$ & $\begin{array}{c}\text { Modelo } 4 \\
\text { QV social }\end{array}$ & $\begin{array}{c}\text { Modelo 5 } \\
\text { QV } \\
\text { ambiental }\end{array}$ \\
\hline & B & $\beta$ & $\boldsymbol{\beta}$ & $\beta$ & $\beta$ \\
\hline Género**** & -0.06 & -0.05 & -0.03 & $-0.14 * *$ & $-0.09 *$ \\
\hline Idade & -0.06 & $-0.13^{* *}$ & $-0.11^{* *}$ & -0.03 & -0.04 \\
\hline Estado civil ${ }^{* * * * *}$ & $-0.15^{* *}$ & $-0.17 * * *$ & $-0.18 * * *$ & $-0.20 * * *$ & $-0.18 * * *$ \\
\hline Escolaridade & $0.14^{* *}$ & $0.16^{* * *}$ & $0.11^{* *}$ & -0.05 & $0.20 * * *$ \\
\hline Sobrecarga de trabalho & 0.07 & 0.04 & 0.03 & -0.02 & 0.11* \\
\hline Baixa Autonomia & -0.05 & -0.04 & $-0.15^{* * *}$ & -0.03 & 0.01 \\
\hline Baixa Transparência de papel & $-0.16^{* *}$ & $-0.15^{* *}$ & $-0.16^{* *}$ & -0.11 & $-0.15^{* *}$ \\
\hline Baixo Suporte chefia & 0.02 & -0.08 & 0.03 & 0.02 & -0.01 \\
\hline Baixa Confiança/ Justiça & 0.03 & 0.08 & -0.01 & -0.04 & -0.04 \\
\hline Gestão do Stress/Burnout & $0.41^{* * *}$ & $0.48^{* * *}$ & $0.49 * * *$ & $0.38^{* * *}$ & $0.42^{* * *}$ \\
\hline
\end{tabular}

Nota. $^{* * *} p<.001 ;{ }^{* *} p<.01 ;{ }^{*} p<.05 ;$ n.s. = não significativo; ${ }^{* * *}(1-$ Feminino; 2 - Masculino $) ;{ }^{* * * *}(1-$ Casado/união facto; 2 - solteiro/divorciado)

No que diz respeito aos reformados com atividade profissional (Tabela 8), salienta-se a importância da escolaridade para a explicação da QV geral e ambiental e a relevância da gestão do stress/burnout para a compreensão da $\mathrm{QV}$ física e psicológica. Os modelos referentes à $\mathrm{QV}$ geral e $\mathrm{QV}$ social não são estatisticamente significativos. 0 modelo referente à QV física apresenta um poder explicativo de $41 \%$ da variância, $F(10.33)=4.03 ; p<0.001$.

0 terceiro modelo (QV psicológica) explica $29 \%$ da variância, $F(10.33)=2.77 ; p<0.01$. Em relação ao modelo cinco $(\mathrm{QV}$ ambiental) que explica $22 \%$ da variância, $F(10.37)=2.30 ; p<0.05$.

Tabela 8. Modelos de regressão linear múltipla para o estudo da qualidade de vida nos indivíduos reformados com atividade profissional

\begin{tabular}{lccccc}
\hline & $\begin{array}{c}\text { Modelo 1 } \\
\text { QV geral }\end{array}$ & $\begin{array}{c}\text { Modelo 2 } \\
\text { QV física }\end{array}$ & $\begin{array}{c}\text { Modelo 3 } \\
\text { QV psicológica }\end{array}$ & $\begin{array}{c}\text { Modelo 4 } \\
\text { QV social }\end{array}$ & $\begin{array}{c}\text { Modelo 5 } \\
\text { QV ambiental }\end{array}$ \\
\cline { 2 - 6 } & $\boldsymbol{\beta}$ & $\boldsymbol{\beta}$ & $\boldsymbol{\beta}$ & $\boldsymbol{\beta}$ & $\boldsymbol{\beta}$ \\
\hline Género**** & -0.05 & 0.18 & $\mathbf{0 . 3 1 *}$ & 0.14 & 0.07 \\
Idade & 0.13 & 0.13 & 0.19 & 0.27 & 0.11 \\
Estado civil***** & -0.21 & -0.19 & 0.02 & -0.03 & -0.07 \\
Escolaridade & $\mathbf{0 . 3 3 *}$ & 0.26 & 0.29 & 0.13 & $\mathbf{0 . 4 3 *}$ \\
Sobrecarga de trabalho & 0.17 & 0.23 & 0.26 & 0.05 & -0.04 \\
Baixa Autonomia & -0.15 & -0.04 & -0.02 & 0.01 & 0.04 \\
Baixa Transparência de papel & -0.21 & $-\mathbf{0 . 3 8 *}$ & -0.22 & -0.40 & -0.27 \\
Baixo Suporte chefia & 0.08 & 0.25 & 0.24 & -0.08 & -0.11 \\
Baixa Confiança/ Justiça & -0.05 & -0.25 & -0.15 & 0.21 & 0.01 \\
Gestão do Stress/Burnout & 0.29 & $\mathbf{0 . 6 5 * * *}$ & $\mathbf{0 . 6 1 * *}$ & 0.28 & 0.08 \\
\hline
\end{tabular}

Nota. $^{* * *} p<.001 ;{ }^{* *} p<.01{ }^{*} p<.05 ;$ n.s. $=$ não significativo; ${ }^{* * * *}(1-$ Feminino; 2 - Masculino $) ;{ }^{* * * *}(1$ Casado/união facto; 2 - solteiro/divorciado)

\section{DISCUSSÃO}

0 presente estudo pretendeu compreender e caracterizar a relação entre os riscos psicossociais e a qualidade de vida e respetivas dimensões. Pretendeu-se, ainda, estudar o impacto do género, idade e situação profissional dos participantes nesta relação.

Os resultados revelam que existe uma relação estatisticamente significativa entre as dimensões da qualidade de vida e dos diferentes riscos psicossociais. Salienta-se a relação entre a capacidade gestão de stress/burnout e a qualidade de vida física e psicológica, e entre a sobrecarga de trabalho e a qualidade de vida física. A saúde e qualidade de vida devem ser compreendidas numa perspetiva biopsicossocial, que envolve a saúde física, psicológica, social, laboral e ambiental. Estes fatores influenciam a expressão e 
experiência de saúde e doença, adaptação e desadaptação, e a saúde e doença podem resultar da interligação destes fatores (Schneider et al., 2005; Taylor, 2012).

Verificamos diferenças de género, sendo que as mulheres apresentam perceção mais negativa em relação qualidade de vida física, psicológica e ambiental, e menor capacidade de gestão de stress/burnout e baixa perceção confiança/justiça. As mulheres frequentemente aparecem como um grupo de risco e diversos estudos apresentam resultados que reforçam o facto de as mulheres apresentarem uma perceção de qualidade de vida e bem-estar mais negativa, assim como perceção de desigualdade de género ao nível das competências e oportunidades (Gholami, et al., 2013).

Os participantes mais velhos, com mais de 60 anos de idade referem uma melhor capacidade de gestão do stress/burnout. Os participantes mais novos, com 60 anos ou menos de idade apresentam uma perceção mais positiva da sua qualidade de vida em termos gerais, assim como na qualidade de vida física, psicológica e social.

Quando se analisam os dados comparando a situação profissional dos participantes, verificamos que de um modo geral, o grupo com uma perceção mais positiva dos riscos psicossociais e da qualidade de vida é o grupo de participantes reformados, mas que mantém uma atividade profissional ativa. A maior preocupação dos participantes com atividade profissional é a capacidade de gestão do stress/burnout e sobrecarga de trabalho. 0 processo de envelhecimento e reforma trazem alterações e desafios psicológicos e sociais, por um lado verifica-se uma redução do stress e pressão pelo exercício de uma profissão (Khamisa, et al.,2015) mas, por outro lado, pode-se verificar uma desvalorização social e a perda de uma identidade relacionada com a profissão (Clark \& Hapson, 2001; Eatough et al., 2012; Gaspar \& Torres, 2015; SAMHSA Advisory, 2013; Nieuwenhuijsen, et al., 2010).0 presente estudo permite compreender a relação da situação profissional (profissionalmente ativo; reformado e reformado com atividade profissional), dos fatores psicossociais e da perceção de QV dos participantes no processo de envelhecimento e reforma. 0 estudo revela que grande parte das dimensões dos fatores psicossociais e a qualidade de vida e respetivas dimensões variam consoante a situação profissional. Os participantes com atividade profissional ativa apresentam menor capacidade de gestão do stress/burnout, maior sobrecarga de trabalho, melhor perceção de qualidade de vida social e ambiental. São os participantes reformados com atividade profissional ativa que apresentam uma melhor perceção de qualidade de vida geral, qualidade de vida física e psicológica, por serem provavelmente aqueles que maior empenhamento afetivo têm no trabalho, não sentido o peso da obrigatoriedade de terem de trabalhar porque a decisão de continuar ativo foi um comportamento de escolha motivado que pode facilitar a transição e ajustamento à reforma total (Kim \& Feldman, 2000).

Analisando a relação de cada um dos riscos psicossociais com cada uma das diferentes dimensões da qualidade de vida, verificamos que, de um modo geral, são as dimensões capacidade de gestão do stress/burnout e sobrecarga de trabalho que mais influência têm na qualidade de vida e respetivas dimensões, especialmente, na qualidade de vida física e psicológica. As dimensões psicológicas apresentam um grande impacto na qualidade de vida, perceção de bem-estar e perceção de competências, no processo de envelhecimento a qualidade de vida física ganha um peso também muito relevante uma vez que o envelhecimento pode estar associado ao aumento de problemas de saúde física com tendência para a cronicidade (Gaspar et al., 2012; Gaspar et al., 2009; Scheibe \& Kuba, 2017).

A prevenção e intervenção no processo de pré-reforma, reforma e envelhecimento da população deve incluir a promoção de competências sociais e psicológicas na população-alvo, na família e comunidade. A promoção de saúde e comportamentos saudáveis em todas as idades contribui para prevenir ou retardar o desajustamento e o desenvolvimento de doenças crónicas ou outras doenças relacionadas ao envelhecimento. Se a doença já existe, é importante minimizar as consequências através da deteção precoce, atendimento de qualidade e promoção de competências psicossociais. A intervenção ao nível da promoção da saúde deve criar ambientes físicos, sociais e laborais que promovam a saúde e a participação de pessoas e é importante mudar as atitudes pessoais e sociais, a fim de incentivar a participação das pessoas no processo de envelhecimento (WHO, 2012).

A avaliação da qualidade de vida e riscos psicossociais, nomeadamente nos últimos anos de atividade profissional, pode ser útil na triagem e identificação de indivíduos que estão em risco de desenvolver comportamentos ou complicações de saúde, o que é crucial para programas sociais, programas de promoção e prevenção da saúde, que em última análise deveriam ser da responsabilidade das organizações. Torna-se relevante compreender a relação entre qualidade de vida, riscos psicossociais e o impacto que a idade, género, e situação profissional podem ter no processo de reforma e envelhecimento. Estes indicadores fornecem um forte argumento para a investigação científica em qualidade de vida e riscos psicossociais na população mais idosa usando uma perspetiva biopsicossocial por psicólogos, gestores de recursos humanos, outros profissionais de saúde / sociais e contextos comunitários que têm que enfrentar o desafio de fornecer serviços sensíveis às diferenças, e mudanças sociais, culturais e individuais, tais como, 
permitir que o mercado de trabalho responda às consequências económicas e sociais do envelhecimento da população, como gerir um número elevado de gerações de trabalhadores com características tão dispares, promovendo a solidariedade entre gerações e locais de trabalho saudáveis e sustentáveis. (Huebner et al., 2004; WHO, 2012): As próprias organizações devem ter responsabilidade, que fornecerá sobretudo aos Psicólogos Organizacionais e aos Gestores de Recursos Humanos a oportunidade de dar contributos que são consistentes com a missão da área científica de Comportamento Organizacional e que tem potencial de elevar a área aos olhos da sociedade.

O estudo apresentou algumas limitações, nomeadamente a representatividade, pois utilizou-se uma amostra nacional de conveniência. Portanto, os resultados não podem ser considerados representativos para a população em estudo. Uma vez que os fatores físicos e psicológicos foram os mais relevantes, pode ser interessante estudar a população com situações de saúde especificas, nomeadamente, doenças crónicas específicas e comorbilidade entre as doenças, incluindo problemas de saúde mental relacionados à demência, depressão e ansiedade, assim como aprofundar situações profissionais, situação profissional, profissão, função, escolaridade entre outras. Idealmente, dever-se-ia conduzir um estudo longitudinal comparando a situação de pré-reforma com a de reforma. Teria, também, interesse aprofundar as razões subjacentes a manter-se ativo mesmo estando reformado, i.e os designados bridge employment.

Apesar das limitações do estudo, os resultados e modelos propostos podem ser considerados contribuições para uma melhor compreensão e promover uma intervenção mais efetiva relacionada ao risco psicossocial, promoção da qualidade de vida e ajustamento no processo de reforma e envelhecimento.

Os resultados sugerem fortemente que ser mulher, ter mais de 60 anos e ser reformado sem atividade profissional podem ser considerados fatores ligados ao risco, e podem prejudicar a perceção de qualidade de vida ou pelo menos levar ao aumento da vulnerabilidade. A capacidade de adaptação e ajustamento aos desafios e mudanças no processo de envelhecimento e o desenvolvimento de competências socioemocionais são fatores ligados à proteção. Numa perspetiva biopsicossocial, este processo de ajuste com enfoque nas oportunidades de um envelhecimento positivo, deve ter o envolvimento global, das pessoas, das famílias, assim como das entidades empregadoras, dos serviços de suporte e políticas de saúde e sociais (Brown, et al., 2010; Miranzi et al., 2008; Odili et al., , 2008).

\section{REFERÊNCIAS}

Adams, G., Garcia, D.M., Purdie-Vaughns, V., \& Steele, C.M. (2006). The detrimental effects of a suggestion of sexism in an instruction situation. Journal of Experimental Social Psychology, 42, 602-615. https://doi.org/10.1016/j.jesp.2005.10.004

Behncke, S. (2012). Does retirement trigger ill health? Health economics, 21(3), 282-300. https://doi.org/10.1002/hec. 1712

Bonde, J. P. E. (2008). Psychosocial factors at work and risk of depression: a systematic review of the epidemiological evidence. Occupational and environmental medicine, 65(7), 438-445. https://doi.org/10.1136/oem.2007.038430

Brown, D., Pleasants, R., Ohar, J., Kraft, M., Donohue, J., Mannino,D., Liao, W. \& Herrick, H. (2010). Healthrelated quality of life and chronic obstructive pulmonary disease in North Carolina. North American Journal of Medical Sciences, 2(2), 60-65. https://doi.org/10.4297/najms.2010.260

Burgard, S. A., \& Lin, K. Y. (2013). Bad jobs, bad health? How work and working conditions contribute to health disparities. American Behavioral Scientist, 57(8), 1105-1127. https://doi.org/10.1177/0002764213487347

Byles, J., Tavener, M., Robinson, I., Parkinson, L., Smith, P. W., Stevenson, D., Leigh, L. \& Curryer, C. (2013). Transforming retirement: New definitions of life after work. Journal of women \& aging, 25(1), 24-44. https://doi.org/10.1080/08952841.2012.717855

Canavarro, M. C., Simões, M. R., Vaz Serra, A., Pereira, M., Rijo, D., Quartilho, M. J., Gameiro, S., Paredes, T., \& Carona, C. (2007). Instrumento de avaliação da qualidade de vida da Organização Mundial de Saúde: WHOQOL-Bref. In M. Simões, C. Machado, M. Gonçalves, \& L. Almeida (Eds.), Avaliação psicológica: Instrumentos validados para a população portuguesa (Vol. III, pp. 77-100). Quarteto Editora.

Clark, M., \& Hampson, S. E. (2001). Implementing a psychological intervention to improve lifestyle selfmanagement in type 2 diabetes. Patient Education and Counseling, 42, 247-256. https://doi.org/10.1016/s0738-3991(00)00128-2

Coe, N. B., \& Zamarro, G. (2011). Retirement effects on health in Europe. Journal of health economics, 30(1), 77-86. https://doi.org/10.1016/j.jhealeco.2010.11.002

Eatough, E. M., Way, J. D., \& Chang, C. H. (2012). Understanding the link between psychosocial work stressors and work-related musculoskeletal complaints. Applied ergonomics, 43(3), 554-563. https://doi.org/ 10.1016/j.apergo.2011.08.009 
Efklides, A. \& Moraitou, D. (2013). Introduction: Looking at quality of life and well-being from a positive psychology perspective. In M. Platsidou, D. Moraitou (Eds) A positive psychology perspective on quality of life (Vol. 51, pp.1-16). Springer Science \& Business Media.

European Commission. (2015). The 2015 Ageing Report - Economic and budgetary projections for the $28 \mathrm{EU}$ Member States (2013-2060). https://ec.europa.eu/economy_finance/publications/european_economy/2015/pdf/ee3_en.pdf

European Commission. (2018). The 2018 Ageing Report - Economic and budgetary projections for the $28 \mathrm{EU}$ Member States (2016-2070). https://ec.europa.eu/info/sites/info/files/economyfinance/ip079_en.pdf

Fehr, R. (2012). Is retirement always stressful? The potential impact of creativity. American Psychologist, 67, 76-77. https://doi.org/10.1037/a0026574

Fonseca, A. M. (2012). Do trabalho à reforma: quando os dias parecem mais longos. Sociologia, Revista da Faculdade de Letras da Universidade do Porto, 2, 75-95.

Friedman, E. M., Ruini, C., Foy, R., Jaros, L., Sampson, H., \& Ryff, C. D. (2017). Lighten UP! A community-based group intervention to promote psychological well-being in older adults. Aging \& mental health, 21(2), 199-205. https://doi.org/10.1080/13607863.2015.1093605

Gaillard, M. \& Desmette, D. (2010). (In)validating Stereotypes About Older Workers Influences Their Intentions to Retire Early and to Learn and Develop. Basic and Applied Social Psychology, 32(1), 8698. https://doi.org/10.1080/01973530903435763

Gaspar, T., Matos, M. G., Ribeiro, J. L., Leal, I., Erhart, M., \& Ravens-Sieberer, U. (2012). Health-related quality of life inchildren and adolescents: Subjective well being. Spanish Journal of Psychology, 15(1), 177186. http://dx.doi.org/10.5209/rev_SJOP.2012.v15.n1.37306

Gaspar, T., Matos, M. G., Ribeiro, J., Leal, I., \& Ferreira, A. (2009). Health-related quality of lifein children and adolescents and associated factors. Journal of Cognitive and Behavioral Psychotherapies, 9, 33-48.

Gaspar, T. \& Torres, I. (2015). Fatores psicossociais e profissionais promotores de qualidade de vida no processo de reforma e envelhecimento ativo. Estudo de envelhecimento ativo. Universidade Lusíada de Lisboa.

Gholami, A., Azini, M., Borji, A., Shirazi, F., Sharafi, Z., \& Zarei, E. (2013). Quality of life in patiets with type 2 diabetes: Application of WHOQOL-BREF scale. Shiraz E-Medical Journal, 14(3),162-171.

Halvorsrud, L. \& Kalfoss, M. (2007). The conceptualization and measurement of quality of life in older adults: a review of empirical studies published during 1994-2006. European Journal of Ageing, 4, 229-246. https://doi.org/10.1007/s10433-007-0063-3

Helkavaara, M., Saastamoinen, P., \& Lahelma, E. (2011). Psychosocial work environment and emotional exhaustion among middle-aged employees. BMC research notes, 4(1), 101. https://doi.org/10.1186/1756-0500-4-101

Huebner, E., Suldo, S., Smith, L., \& McKnight, C. (2004). Life satisfaction in children and youth:Empirical foundations and implications for school psychologists. Psychology in the Schools, 41, 81-93. https://doi.org/10.1002/pits.10140

Illario, M., Vollenbroek-Hutten, M. M., Molloy, D. W., Menditto, E., Iaccarino, G., \& Eklund, P. (2016). Active and healthy ageing and independent living 2016. Journal of aging research, 2016. https://doi.org/10.1155/2016/8062079

Jönsson, S. (2012). Psychosocial work environment and prediction of job satisfaction among Swedish registered nurses and physicians-a follow-up study. Scandinavian Journal of Caring Sciences, 26(2), 236-244. https://doi.org/10.1111/j.1471-6712.2011.00924.x

Khamisa, N., Oldenburg, B., Peltzer, K., \& Ilic, D. (2015). Work related stress, burnout, job satisfaction and general health of nurses. International journal of environmental research and public health, 12(1), 652-666. https://doi.org/10.3390/ijerph120100652

Kim, S., \& Feldman, D. C. (2000). Working in retirement: The antecedents of bridge employment and its consequences for quality of life in retirement. Academy of Management Journal, 43, 1195-1210. https://doi.org/10.2307/1556345

Kim, J. E., \& Moen, P. (2002). Retirement transitions, gender, and psychological well-being a life-course, ecological model. Journal of Gerontology, series B: Psychological Science and Social Science, 57(3), 212-222. https://doi.org/10.1093/geronb/57.3.P212

Koch S.C., Müller, S.M., Sieverding, \& M. (2008). Women and computers: Effects of stereotype threat on attribution of failure. Computers \& Education, 51, 1795-1803. https://doi.org/10.1016/j.compedu.2008.05.007

Kristensen, T., Hannerz, H., Hogh, A. \& Borg, V. (2005). The Copenhagen psychosocial Questionnaire - a tool for the assessment and improvement of the psychosocial work environment. Scandinavian Journal of Work, Environment and Health, 31(6), 438-449. https://doi.org/10.5271/sjweh.948 
Kuba, K., \& Scheibe, S. (2017). Let it be and keep on going! Acceptance and daily occupational well-being in relation to negative work events. Journal of Occupational Health Psychology, 22(1), 59-70. http://dx.doi.org/10.1037/a0040149

Kulik, C., Perera, S. \& Cregan, C. (2016). Engage me: The mature-age worker and stereotype threat. The Academy of Management Journal, 59(6), 1-57. https://doi.org/10.5465/amj.2015.0564

Malinen, S. \& Johnston, L. (2013). Workplace ageism: discovering hidden bias. Experimental Aging Research, 39, 445-465. https://doi.org/10.1080/0361073X.2013.808111

Mari, F. R., Alves, G. G., Aerts, D. R. G. S. \& Camara, S. (2016). O processo de envelhecimento e a saúde: o que pensam as pessoas de meia-idade sobre o tema. Revista Brasileira de Geriatria e Gerontologia, 19(1), 35-44. http://dx.doi.org/10.1590/1809-9823.2016.14122

Miranzi, S. D. S. C., Ferreira, F. S., Iwamoto, H. H., Pereira, G. D. A. \& Miranzi, M. A. S. (2008). Qualidade de vida de indivíduos com diabetes mellitus e hipertensão acompanhados por uma equipe de saúde da família. Texto Contexto Enfermagem, 17(4), 672. https://doi.org/10.1590/S010407072008000400007

Nieuwenhuijsen, K., Bruinvels, D., \& Frings-Dresen, M. (2010). Psychosocial work environment and stressrelated disorders, a systematic review. Occupational medicine, 60(4), 277-286. https://doi.org/10.1093/occmed/kqq081

Odili, V., Ugboka, L., \& Oparah, A. (2008). Quality of life of people with diabetes in Benin City as measured with WHOQOL-BREF. Internet J Law, Healthc Ethics, 6(2).

Rabl, T. (2010). Age, discrimination, and achievement motives: A study of German employees. Personnel Review, 39(4), 448-467. https://doi.org/10.1108/00483481011045416

SAMHSA Advisory. (2013). Diabetes care for clients in behavioral health treatment. Fall, 12(1), 1-11.

Schneider, W., Gruman, J., \& Coutts, L. (2005). Applied social psychology: Understanding and addressing social and practical problems. Sage.

Silva, C., Amaral, V., Pereira, A., Bem-haja, P., Pereira, A., Rodrigues, V., Cotrim, V., Silvério, T., \& Nossa, P. (2011). Copenhagen Psychosocial Questionnaire. Portugal e Países Africanos de Língua Oficial Portuguesa. Departamento de Educação, Universidade de Aveiro.

Smith J.L., Sansone, C., \& White, P.H. 2007. The stereotyped task engagement process: The role of interest and achievement motivation. Journal of Educational Psychology, 99(1), 99-114. https://doi.org/10.1037/0022-0663.99.1.99

Steptoe, A., Deaton, A., \& Stone, A. A. (2015). Subjective wellbeing, health, and ageing. The Lancet, 385(9968), 640-648. https://doi.org/10.1016/ S0140-6736(13)61489-0

Taylor, S. E. (2012). Health Psychology (8th ed.). McGraw Hill.

Teles, M. A. B., Barbosa, M. R., Vargas, A. M. D., Gomes, V. E., Ferreira, E. F., de Barros Lima, A. M. E., \& Ferreira, R. C. (2014). Psychosocial work conditions and quality of life among primary health care employees: A cross sectional study. Health and Quality of Life Outcomes, 12(1), 72. https://doi.org/10.1186/1477-7525-12-72

Theorell, T., Hammarström, A., Aronsson, G., Bendz, L. T., Grape, T., Hogstedt, C., Marteinsddottir, I., Skoog, I. \& Hall, C. (2015). A systematic review including meta-analysis of work environment and depressive symptoms. BMC public health, 15(1), 738. https://doi.org/10.1186/s12889-015-1954-4

Truxillo, D. M., McCune, E. A. \& Bertolino, M. (2012). Perceptions of older versus younger workers in terms of big five facets, proactive personality, cognitive ability and job performance. Journal of Applied Social Psychology, 42(11), 2607-2639. https://doi.org/10.1111/j.1559-1816.2012.00954.x

van der Heide, I., van Rijn, R. M., Robroek, S. J., Burdorf, A., \& Proper, K. I. (2013). Is retirement good for your health? A systematic review of longitudinal studies. BMC public health, 13(1), 1180. https://doi.org/10.1186/1471-2458-13-1180

Wang, M. (2007). Profiling retirees in the retirement transition and adjustment process: examining the longitudinal change patterns of retirees' psychological well-being. Journal of Applied Psychology, 92, 455-474. https://doi.org/10.1037/0021-9010.92.2.455

Wang, M., Henkens, K. \&, van Solinge, H. (2011). Retirement adjustment: a review of theoretical and empirical advancements. American Psychologist, 66(3), 204-213. https://doi.org/10.1037/a0022414

World Health Organization. (2002). The World Health Report 2001. Mental Health: New Understanding, New Hope. https://www.who.int/whr/2001/en/whr01_en.pdf?ua=1

World Health Organization. (2012). Good health adds life to years: Global brief world health 2012. https://apps.who.int/iris/bitstream/handle/10665/70853/WHO_DCO_WHD_2012.2_eng.pdf;jsess ionid=644E60BD52D2ABE1420DB76B0EA38242? sequence $=1$ 
World Health Organization (WHO). (2015). World report on ageing and health. https://apps.who.int/iris/bitstream/handle/10665/186463/9789240694811_eng.pdf?sequence= 1

Wong, J. Y., and Earl, J. K. (2009). Towards an integrated model of individual, psychosocial, and organizational predictors of retirement adjustment. Journal of Vocational Behaviour. 75, 1-13. https://doi.org/10.1016/j.jvb.2008.12.010

$\begin{array}{lr}\text { Historial do artigo } \\ \text { Recebido } & 05 / 2019 \\ \text { Aceite } & 07 / 2020 \\ \text { Publicado } & 08 / 2020\end{array}$

